To be published in Journal of Optical Communications and Networking:

Title: $\quad$ Fragmentation-Aware Algorithm with Bordering Super-Channels in Spectrally/Spatially-Flexible Optical Networks

Authors: Piotr Lechowicz,Massimo Tornatore,Adam Wodarczyk,Krzysztof Walkowiak

Accepted: 06 March 20

Posted 09 March 20

DOI: $\quad$ https://doi.org/10.1364/JOCN.382838

(C) 2020 Optical Society of America 


\title{
Fragmentation Metrics and Fragmentation-Aware Algorithm for Spectrally/Spatially-Flexible Optical Networks
}

\author{
Piotr Lechowicz ${ }^{1}$, Massimo Tornatore $^{2}$, Adam WŁodarczYK ${ }^{1}$, And Krzysztof Walkowiak ${ }^{1}$ \\ ${ }^{1}$ Department of Systems and Computer Networks, Wrocław University of Science and Technology, Poland \\ ${ }^{2}$ Department of Electronics, and Bioengineering, Politecnico di Milano, Italy \\ ${ }^{*}$ Corresponding author: piotr.lechowicz@pwr.edu.pl
}

Compiled March 6, 2020

Spectrally/spatially-flexible optical networks (SS-FONs) are a promising solution to cope with future traffic requirements in optical backbone networks. SS-FONs exploit the spatial dimension to increase network capacity while preserving resource management flexibility, as they still operate within a flex-grid composed of small frequency slots (slices). Flex-grid allows realizing transmission using super-channels (SChs) that comprise a set of contiguous slots. In this paper, we focus on spectral SChs, i.e., a SCh realized only on one spatial mode on each fiber. In SS-FONs, setting up and tearing down multiple lightpath requests within flex-grid may result in spectrum fragmentation, and, in turn, blocking of requests. In this work, we investigate several fragmentation metrics in SS-FON. The problem of identifying appropriate metrics to measure fragmentation has been investigated in single-core elastic optical networks (EONs), but, to the best of our knowledge, there is lack of such investigation for SS-FONs. Therefore, we propose several fragmentation metrics for SS-FON. We introduce the concept of bordering super-channels (B-SChs), i.e., SChs whose spectrum is allocated at the "border" of already allocated spectrum slots that are promising SChs to be chosen to minimize fragmentation. The investigation of all candidate B-SCh for a request allows us to find the one which minimizes network fragmentation, and, in turn spectrum waste. Hence, we propose a fragmentation-aware algorithm with bordering super-channels (FA-BSC) that assigns optical resources to dynamic requests utilizing information from the proposed fragmentation metrics and the set of candidate B-SChs. Experiments on a representative network topology show that the investigation of multiple B-SChs in the fragmentation-aware algorithm reduces the blocking probability when compared to the reference fragmentation-aware algorithms. Finally, we analyze the impact of a spatial continuity constraint on the network fragmentation. ๑) 2020 Optical Society of America

http://dX.doi.org/10.1364/ao.XX.XXXXXX

\section{INTRODUCTION}

The ever-increasing Internet traffic keeps fueling the growth of bandwidth demand in optical networks. Spectrally/spatiallyflexible optical networks (SS-FONs) are considered a promising direction to accommodate the growing traffic [1, 2].

SS-FON is a parallel application of two orthogonal technologies - space-division multiplexing (SDM) and elastic optical networks (EONs). SDM provides significant capacity increase by exploiting the spatial dimension and enabling parallel transmission by co-propagation of an optical signal in suitably designed optical fibers. Examples of SDM fibers are bundles of single-core single-mode fibers (SMFBs), multi-core fibers (MCFs), few-mode fibers (FMFs) and few-mode multi-core fibers (FM-MCF). Without loss of generality, the term "spatial mode" refers to a single resource in a spatial dimension regardless of the selected fiber technology, e.g., fiber, core, mode or group of modes $[2,3]$.

EONs represent an evolution of traditional Wavelength Division Multiplexing (WDM) networks. EONs operate within a flexible (elastic) grid divided into small frequency slots (slices) and they can assign the proper amount of optical bandwidth to serve different types of bandwidth requests. The optical spectrum can be divided and assigned to different requests using optical spectral corridors called super-channels (SChs) that can comprise an arbitrary set of contiguous slots [4].

A combination of both SDM and EON technology in SS-FON allows assigning bandwidth to requests using spectral, spatial or joint spectral/spatial SChs, depending on the applied fibers and nodes architecture $[2,3]$. In this work, we consider only 
spectral SChs, where on a particular link, a super-channel can be carried over multiple contiguous spectral resources but over only one spatial mode (i.e., on any link, the SCh cannot be split over multiple spatial modes). Analysis of spectral/spatial SChs is left for future work..

In SS-FONs, setting up and tearing down dynamic requests may result in spectrum fragmentation. When spectrum becomes fragmented, available resources are divided into small free spectral intervals, which are unlikely to be utilized by future requests and may increase blocking $[5,6]$. A key issue in SS-FONs is spatial continuity constraint (SCC), i.e., the constraint that a unique spatial mode shall be assigned to all the channels along a lightpath. SCC is enforced in most of the currently proposed SS-FON architectures, e.g., in the case of independent switching over SMFBs, or the case of fractional joint switching over FM-MCFs [7-9]. In particular, SCC enforcement is required when SDM switching devices without a sufficient amount of internal spatial switching capability are used (e.g., the reconfigurable optical add-drop multiplexers for independent switching without lanechange support in [8]). These devices have lower complexity, and hence lower cost, but they are also characterized by limited or no switching flexibility in the spatial dimension $[3,10]$. Thus, in SS-FONs, it is important to investigate the impact of SCC on fragmentation and blocking probability when compared to the case when SCC is not enforced [11].

Most conventional routing, spectrum, and space assignment algorithms (RSSA) proposed as of today apply first-fit (or other) policy for selecting candidate super-channels on each candidate routing path, as an investigation of all possible SCh candidates is extremely challenging in terms of the time-complexity. Thus, new methods that effectively explore the solution space for SCh assignment are needed.

In this work, the contribution is threefold. First, we propose several new fragmentation metrics tailored for SS-FONs. Note that, while various fragmentation metrics have been proposed for classical EONs (i.e., without SDM) [5], our study is the first one, to the best of our knowledge, to investigate fragmentation metrics in SS-FONs. Second, we introduce the concept of bordering super-channels (B-SChs), i.e., SChs whose spectrum is at the "border" of already allocated spectrum slots with the intent of minimizing spectrum waste, and hence, fragmentation. Next, we prove that the B-SCh that yields the lowest fiber fragmentation (at least for two fragmentation metrics) can be optimally identified. We propose a method for finding such B-SChs, and extend this concept to find SChs on routing paths for cases with and without SCC. Finally, we propose a fragmentation-aware RSSA algorithm with bordering super-channels (FA-BSC) that leverage B-SChs to minimize the bandwidth blocking probability (BBP). Our numerical results confirm that the introduction of intelligent B-SCh selection decreases the proposed network fragmentation metrics compared to baseline methods which consider either first-fit SCh selection policy or best-fit policy from the set of randomly selected SChs.

The rest of the paper is organized as follows. In Sec. 2, we discuss related works. In Sec. 3, our SS-FON model is described and we introduce the new fragmentation metrics for SS-FONs. In Sec. 4, we elaborate on the concept of bordering super-channels. Then, in Sec. 5, we describe our proposed fragmentation-aware algorithms. In Sec 6, illustrative numerical experiments are presented. In Sec. 7, we conclude our study. Tab. 1 presents lists of symbols used in the paper.
Table 1. List of Symbols

\begin{tabular}{|c|c|}
\hline$\lfloor a / b\rfloor$ & integer division $a$ over $b$ \\
\hline$B$ & bandwidth on each link \\
\hline $\operatorname{BSCh}\left(e, k, n_{d p}\right)$ & $\begin{array}{l}\text { set of bordering super-channels of size } n_{d p} \text { on spa- } \\
\text { tial mode } k \text { on link } e\end{array}$ \\
\hline$B S C h_{n o S C C}$ & $\begin{array}{l}\text { set of candidate bordering super-channels for path } \\
p \text { of size } n_{d p} \text { when SCC is relaxed }\end{array}$ \\
\hline$B S C h_{S C C}\left(p, n_{d p}\right)$ & $\begin{array}{l}\text { set of candidate bordering super-channels for path } \\
p \text { of size } n_{d p} \text { when SCC is assumed }\end{array}$ \\
\hline$D$ & set of requests \\
\hline E & set of unidirectional physical network links \\
\hline$E(p)$ & set of links included in path $p$ \\
\hline$F_{\text {link }}^{m}(e)$ & link $e$ fragmentation calculated using metric $m$ \\
\hline & network fragmentation calculated using metric $m$ \\
\hline$F_{s m}^{m}(e, k)$ & $\begin{array}{l}\text { spatial mode } k \text { fragmentation of link } e \text { calculated } \\
\text { using metric } m\end{array}$ \\
\hline$f_{i}^{A N D}$ & A-Fiber for fiber $i$ \\
\hline$f_{p}^{O R, n}$ & $\begin{array}{l}\text { fiber representing path } p(\mathrm{FREP}) \text { when } \mathrm{SCC} \text { is re- } \\
\text { laxed }\end{array}$ \\
\hline$f_{p}^{O R}$ & $\begin{array}{l}\text { fiber representing path } p(\mathrm{FREP}) \text { when } \mathrm{SCC} \text { is as- } \\
\text { sumed }\end{array}$ \\
\hline$f_{p, k}^{O R, n}$ & $\begin{array}{l}\text { spatial mode } k \text { of fiber representing path } p \text { (FREP) } \\
\text { when SCC is relaxed }\end{array}$ \\
\hline$f_{p, k}^{O R, S C C}$ & $\begin{array}{l}\text { spatial mode } k \text { of fiber representing path } p \text { (FREP) } \\
\text { when SCC is assumed }\end{array}$ \\
\hline G & $\begin{array}{l}\text { set of available SCh granularities including guard- } \\
\text { band }\end{array}$ \\
\hline $\mathcal{G}(V, E)$ & directed graph \\
\hline$\Gamma(e, k)$ & set of free segments on spatial mode $k$ on link $e$ \\
\hline$\left|\gamma_{e k}\right|$ & size of free segment $\gamma_{e k} \in \Gamma(e, k)$ \\
\hline K & set of spatial modes on each link \\
\hline$n_{d p}$ & $\begin{array}{l}\text { number of required slots for request } d \text { on path } p \\
\text { (using the most spectrally-effiecient available mod- } \\
\text { ulation format) }\end{array}$ \\
\hline$P$ & set of candidate routing paths \\
\hline$S$ & set of slots, numbered $1, \ldots,|S|$ \\
\hline$\overline{s_{e k}}$ & slots represented as a binary array \\
\hline$s_{\max }$ & highest allocated slot index in the network \\
\hline$s_{e k}^{\max }$ & $\begin{array}{l}\text { highest allocated slot index on spatial mode } k \text { of } \\
\text { link } e\end{array}$ \\
\hline$S C h(e, k)$ & set of available SChs on spatial mode $k$ on link $e$ \\
\hline $\operatorname{SCh}\left(e, k, n_{d p}\right)$ & $\begin{array}{l}\text { set of available SChs of size } n_{d p} \text { on spatial mode } k \\
\text { on link } e\end{array}$ \\
\hline sch $_{\text {start }}$ & starting slot index of a SCh sch \\
\hline sch $_{\text {end }}$ & ending slot index of a SCh sch \\
\hline$V$ & OH Hethork ilues \\
\hline
\end{tabular}

\section{RELATED WORKS}

Network fragmentation has been widely studied in EONs (see survey papers [5, 12]), while it is only considered in a few papers in the case of SS-FONs. Ref. [13] demonstrated a core classification method for multi-core fibers (MCFs), where each fiber core is dedicated to realize requests of a certain size. A similar approach was presented in [14], where prioritized spectrum areas are used. Each area is used to realize requests of a given size, with the difference that these areas are now created in the frequency domain instead of assigning dedicated spatial resources. Ref. [15] developed a fragmentation-aware algorithm with congestion avoidance that minimizes the number of contiguous spectrum cuts for EONs where spatial elasticity is exploited in the spatial mode by using orbital angular momentum. Authors in [16] considered a cross-core virtual concatenation scheme to minimize the fragmentation in SDM-EONs. Ref. [17] proposed a proactive fragmentation-aware routing in SDM-EONs where the defragmentation process is controlled and triggered by a spectrum-compactness metric. In [18], a dynamic advanced re- 
source reservation was used, where some of the requests have to be realized immediately, and others may be delayed before the setup. The available spectrum is divided into two partitions, each one for a corresponding class of requests. In [19], a proactive fragmentation-aware routing, modulation format, core, and spectrum allocation algorithm was proposed for MCFs that considers spectrum fragmentation state and potential bottlenecks. To avoid bottlenecks, a closeness-centrality metric is introduced to favor selection of paths that contain nodes that contribute less to the formation of bottleneck links. Multipath routing is considered in [20], where a core classification and fragmentation measure metric is used. Ref. [21] showed that quasi-hitless software-defined defragmentation is possible in SDM based on the core switching.

To the best of our knowledge, even though several fragmentation metrics have been proposed in EON (e.g., external fragmentation, Shannon entropy or access blocking probability $[5,12,22])$, there is lack of investigation on the definition of network fragmentation metrics in SS-FONs, except our recent work [11]. SS-FONs introduce the additional spatial dimension, and it should be addressed in the fragmentation metrics. In some forms of SS-FON, the SCC can be relaxed to allow higher flexibility at the expense of higher switching device complexity.

The above-mentioned papers have not considered the impact of SCC on the network fragmentation and BBP. In our recent work [11], we studied various fragmentation metrics for SSFONs with and without SCC, showing that relaxing SCC reduces blocking probability, and as a consequence, allows the allocation of more traffic in the network.

This work is an extension of [11], which was a top-scored paper at ONDM 2019. The main novelties in this work compared to [11] are the introduction of B-SChs concept, the proposal of the FA-BSC fragmentation-aware algorithm that leverages this concept, and evaluation of the algorithm's performance considering SS-FON with and without SCC. Conducted experiments show that FA-BSC minimizes the network fragmentation when compared to the reference fragmentation-aware algorithms. To the best of our knowledge, this is the first paper, that proposes fragmentation improvement by investigation of bordering superchannels in SS-FONs.

\section{FRAGMENTATION METRICS}

In this section, we present the SS-FON model and the notation required to define fragmentation metrics. Then we introduce several fragmentation metrics (many of them are adapted from EONs) to evaluate link fragmentation in SS-FONs [11], namely, External Fragmentation (EF), Shannon Entropy (SE), access blocking probability $(A B P)[5,12,22]$, root of sum of squares (RSS), root mean square factor (RMSF) [23].

\section{A. Network Model}

A SS-FON is modeled as a directed graph $\mathcal{G}(V, E)$ where $V$ is the set of network nodes and set $E$ is the set of unidirectional network links. Each network link $e \in E$ contains a set of spatial modes K. Spatial modes may refer to multiple single-mode fibers, cores, modes, or groups of modes, depending on the considered technology. Each spatial mode $k \in K$ provides $B \mathrm{THz}$ of available spectrum divided into small frequency slots (slices) of $12.5 \mathrm{GHz}$ width, included in set $S$. As in [24], we assume that the network is equipped with coherent transceivers, each operating at fixed baud-rate of 37.5 GBaud and occupying 3 frequency slots. The bit-rate and transmission reach supported by a transceiver depends on the selected modulation format (MF), and are presented in Table 2 [25]. Transmission can be realized using super-channels (SChs), i.e., optical corridors created by grouping several adjacent slots and assigning several adjacent transceivers accordingly. Adjacent SChs are separated using guardbands, each of 1 slot $(12.5 \mathrm{GHz})$ width. The network realizes dynamic requests $D$ and for each one a set of $k$ shortest candidate routing paths (denoted as set $P$ ) is precomputed using Yen's algorithm. The number of required slots for request $d \in D$ on path $p$ is denoted as $n_{d p}$, assuming the most spectrally efficient MF that supports given path length. Possible super-channel granularities depend on the transceiver model, and occupy $3 n+1$ slots, where $n \in \mathcal{N}^{+}$. We assume that the maximum possible requested bit-rate is equal to 1 Tbps, thus $n \leq 20$ when BPSK modulation format is used [6]. For the sake of simplicity, inter-core crosstalk (IC-XT) impairments are not considered (note that the presented model can be extended to include them using worst-case IC-XT estimation as in [6]).

Table 2. Transmission reach and supported bit-rate for MFs.

\begin{tabular}{l|cccc}
\hline & BPSK & QPSK & 8-QAM & 16-QAM \\
\hline $\begin{array}{l}\text { Transmission } \\
\text { reach }[\mathrm{km}]\end{array}$ & 6300 & 3500 & 1200 & 600 \\
\begin{tabular}{l} 
Bit-rate $[\mathrm{Gbps}]$ \\
\hline
\end{tabular} & 50 & 100 & 150 & 200 \\
\hline
\end{tabular}

\section{B. Notation}

Let $\Gamma(e, k)$ denote the set of free segments, i.e., block of contiguous slots, on each spatial mode $k \in K$ of each link $e \in E$. The size of each free segments is defined as $\left|\gamma_{e k}\right|$ for each $\gamma_{e k} \in \Gamma(e, k)$. Let $G$ denote the set of available SCh granularities including guardband according to the transceiver model introduced above. The $s_{e k}^{\max }$ represents the highest allocated slot index on spatial mode $k$ of each $e$. Let $s_{\max }$ denote the highest allocated slot index in the network. Finally, $\lfloor a / b\rfloor$ is an integer division $a$ over $b$.

\section{Network Fragmentation}

Let $F_{\text {net }}^{m}$ denote the whole network fragmentation, which is calculated as an average of links' fragmentation $F_{\text {link }}^{m}(e)$ using the fragmentation metric $m$.

$$
F_{n e t}^{m}=\frac{\sum_{e \in E} F_{\text {link }}^{m}(e)}{|E|} \cdot \frac{s_{\text {max }}}{|S|}
$$

The introduction of the additional spatial dimension in SSFONs does not allow the application of EONs' link fragmentation metrics directly. Therefore, the metric $F_{\text {link }}^{m}(e)$ for each link $e \in E$ is defined as an average of EONs' metric evaluated for each spatial mode $k \in K$ of a given link $e \in E$, denoted as $F_{s m}^{m}(e, k)$.

$$
F_{\text {link }}^{m}(e)=\frac{\sum_{k \in K} F_{s m}^{m}(e, k)}{|K|}
$$

With all presented metrics, a higher number implies more fragmentation.

\section{Metrics}

In this section, we introduce the fragmentation metrics for SSFON. External fragmentation $(E F)$ is a metric originated in computer memory management (Eq. (3)). EF depends on the ratio of the size of the largest free segment on each spatial mode to the total number of available slots. In more detail, EF decreases 
Table 3. Qualitative comparison of fragmentation metrics.

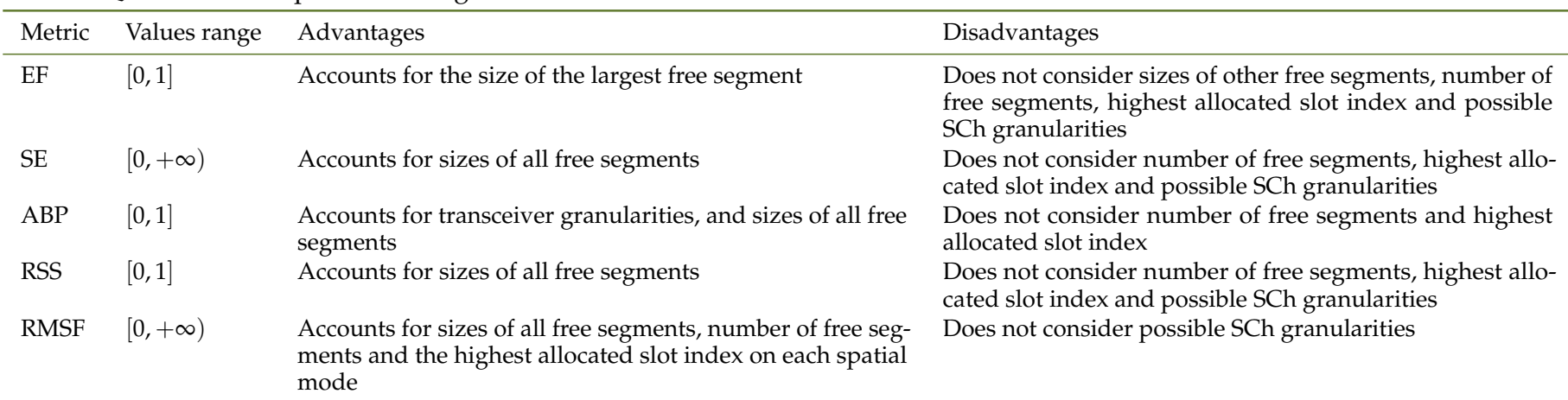

when the size of the largest free segment increases, however, it does not account for the size of other free segments. As a consequence, the comparison of two links using this metric may not be always meaningful due to the differences in fragmentation of smaller free segments $[5,12]$.

$$
F_{e}^{E F}=1-\frac{1}{|K|} \cdot \sum_{k \in K} \frac{\max _{\gamma_{e k} \in \Gamma(e, k)}\left|\gamma_{e k}\right|}{\sum_{\gamma_{e k} \in \Gamma(e, k)}\left|\gamma_{e k}\right|}
$$

Shannon entropy (SE) is applied in information theory to calculate the amount of information available in a message (Eq. (4)). Contrary to EF, SE considers the sizes of all free segments, where a logarithmic function is applied for the inversion of each free segment size. Thus, SE favors creating a small number of large free segments instead of a large number of small segments $[5,12,22,26,27]$.

$$
F_{e}^{S E}=\frac{1}{|K|} \cdot \sum_{k \in K} \sum_{\gamma_{e k} \in \Gamma(e, k)} \frac{\left|\gamma_{e k}\right|}{|S|} \cdot \ln \frac{|S|}{\left|\gamma_{e k}\right|}
$$

Access blocking probability $(A B P)$, in addition to considering the size of each free segment, also accounts for transceiver granularities (Eq. (5)). More specifically, for each SCh granularity $g \in G$, ABP checks how many SChs may be created simultaneously in given free segments and divides this number by the amount of SChs which may be created when all free segments would be combined creating one large free segment. Compared to other metrics, ABP is aware of possible sizes of SChs that can be created in the network $[5,12]$.

$$
F_{e}^{A B P}=1-\frac{1}{|K|} \cdot \sum_{k \in K} \frac{\sum_{\gamma_{e k} \in \Gamma(e, k) \sum_{g \in G}\left\lfloor\left|\gamma_{e k}\right| / g\right\rfloor}}{\sum_{g \in G}\left\lfloor\left(\sum_{\gamma_{e k} \in \Gamma(e, k)}\left|\gamma_{e k}\right|\right) / g\right\rfloor}
$$

Root of sum of squares (RSS) is another possible metric (Eq. (6)). RSS favors larger free segments by taking the square root of the sum of sizes of free segments raised to the power of two. Thus, this metric reflects changes in sizes of smaller free segments, similar to SE and ABP.

$$
F_{e}^{R S S}=1-\frac{1}{|K|} \cdot \sum_{k \in K} \frac{\sqrt{\sum_{\gamma_{e k} \in \Gamma(e, k)}\left(\left|\gamma_{e k}\right|^{2}\right)}}{\sum_{\gamma_{e k} \in \Gamma(e, k)}\left|\gamma_{e k}\right|}
$$

Root mean square factor (RMSF) is presented in Eq. (7), defined as a function of the highest allocated slot index on each spatial mode $s_{e k}^{m a x}$, number of free segments $|\Gamma(e, k)|$ and their sizes. Larger free segments are favored by applying the square root of sum of sizes of free segments raised to the power of two. RMSF metric increases when i) highest slot allocated in each spatial mode increases; ii) number of free segments increases on each spatial mode; iii) the size of smaller segments increases at the cost of decreasing the size of larger ones [23].

$$
F_{e}^{R M S F}=\frac{1}{|K|} \cdot \sum_{k \in K} \frac{s_{e k}^{\max } \cdot|\Gamma(e, k)|}{\sqrt{\frac{\sum_{\gamma_{e k} \in \Gamma(e, k)}\left|\gamma_{e k}\right|^{2}}{|\Gamma(e, k)|}}}
$$

Note, that for metrics EF, ABP and RSS, to avoid division by 0 , if no free segments are available on a spatial mode, the value inside the sum is replaced with 1 (i.e., the highest possible value, as values within the sum are in the range $[0,1])$. For the RMSF metric, such a value is replaced with 0 (i.e., the lowest possible value, as the values within the sum are in the range $[0,+\infty))$. In each case, the idea is to set the fragmentation metric to 0 when there are no available slots in the fiber. Table 3 summarizes the metrics' advantages, disadvantages, and their set of values.

\section{BORDERING SUPER-CHANNELS}

Each spatial mode $k \in K$ of each link $e \in E$ contains slots represented as a binary array $\overline{s_{e k}}$. Each slot is either free or occupied. The status of $i$-th slot is represented as:

$$
\overline{s_{e k}}[i]=\left\{\begin{array}{l}
1, \text { if } i \text {-th slot of } \overline{s_{e k}} \text { is allocated } \\
0, \text { if } i \text {-th slot of } \overline{s_{e k}} \text { is free }
\end{array} \quad \text {, for } i \in 1, \ldots,|S|\right.
$$

Let $S C h(e, k)$ denote a set of available super-channels in the spatial mode $k$ of link $e$. Each $s c h \in S C h(e, k)$ is defined by its starting and ending slot index, denoted as sch start and $s c h_{\text {end }}$ respectively, and can be uniquely described on a given link and spatial mode as a tuple $\left(s c h_{\text {start }}, s_{\text {end }}\right)$. A bordering superchannel $(B-S C h)$ is a SCh whose starting or ending slot index is adjacent to an occupied slot or it is adjacent to any end of the spectrum. In particular, when the starting slot of a SCh is adjacent to the occupied slot or lower end of the spectrum, we call such SCh a left-bordering SCh. Conversely, when the ending slot of a SCh is adjacent to an occupied one or upper end of the spectrum, it is a right-bordering SCh. Finally, when SCh is adjacent to occupied slots at both ends, it is a left-right-bordering SCh. Let $S C h\left(e, k, n_{d p}\right)$ denote a set of available super-channels of size $n_{d p}$ on spatial mode $k \in K$ on link $e \in E$. A set of B-SChs $\operatorname{BSCh}\left(e, k, n_{d p}\right)$ is a subset of $\operatorname{SCh}\left(e, k, n_{d p}\right)$ defined as:

$$
\begin{array}{r}
\operatorname{BSCh}\left(e, k, n_{d p}\right)=\left\{\operatorname{sch} \in \operatorname{SCh}\left(e, k, n_{d p}\right): \overline{s_{e k}}\left[\operatorname{sch}_{\text {start }}-1\right]=1\right. \\
\left.\vee s c h_{\text {start }}=1 \vee \overline{s_{e k}}\left[\operatorname{sch}_{\text {end }}+1\right]=1 \vee \operatorname{sch}_{\text {end }}=|S|\right\}
\end{array}
$$


Figure 1 presents a possible set of B-SChs in single spatial mode $k_{1}$ of a fiber. Note, in this example guardbands are not considered. Gray squares represent occupied slots, while the white squares are the free ones. Let us assume that we want to find B-SChs of 3 slots (hatched gray squares). There are 5 possible locations, as described by the arrows and numbers. The starting and ending slot indices of all B-SChs are $\left\{\left(s_{4}, s_{6}\right),\left(s_{7}, s_{9}\right),\left(s_{15}, s_{17}\right),\left(s_{20}, s_{22}\right),\left(s_{21}, s_{23}\right)\right\}$.

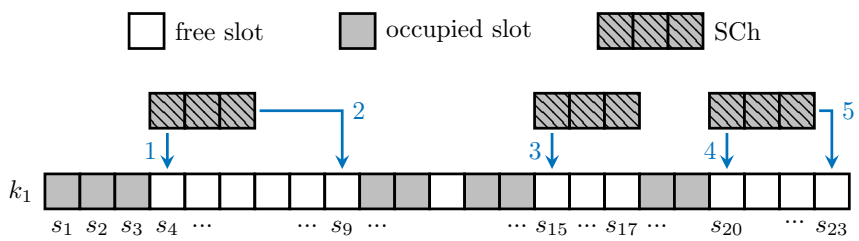

Fig. 1. Possible bordering super-channels in a single spatial mode of a fiber (guardbands are not considered).

\section{A. Impact of Bordering SChs on Spatial Mode Fragmentation}

Let us assume that we have to allocate an SCh of size $n_{d p}$ on spatial mode $k \in K$ of link $e \in E$. Checking all B-SChs should assure that a channel which minimizes the fragmentation metric is found. Since each fragmentation metric is different, it is not possible to claim that it is sufficient to check bordering channels to find the minimum fiber fragmentation for all metrics. Nevertheless, one may claim that the metric which does not have a low fragmentation on one/some of the B-SChs is not designed correctly. The reasoning is that the fragmentation metric should favor creating larger spaces instead of dividing free contiguous slots into smaller spaces. To support this claim, we prove that both metrics - RMSF and ABP - yield lowest spatial mode (and in consequence fiber) fragmentation when B-SChs are considered.

Lemma 4.1. To find SCh of size $n_{d p}$ which minimizes fragmentation metric RMSF on given spatial mode $k \in K$ of link e $\in E$, it is sufficient to check all (and only) bordering SChs of that size, and select the one which minimizes the fragmentation metric the most.

Proof. Assume that the spatial mode is initially divided into $\Gamma(e, k)$ free segments. Each candidate SCh of size $n_{d p}$ in that spatial mode is either bordering or non-bordering one. To prove that the SCh which yields lowest fragmentation is within a set of bordering SChs, it is sufficient to show that for each nonbordering SCh, there exists a bordering SCh which results in a lower fragmentation metric. Moreover, each non-bordering SCh may be associated and compared with the nearest left-bordering SCh.

To prove that, firstly, let us select one of the left-B-SCh $s c h^{\prime}$ in available segments $\Gamma(e, k)$ which is not right-bordering at the same time. Let us assume that the $s c h^{\prime}$ is located in a $\gamma_{e, k} \in \Gamma(e, k)$ segment. As a consequence, the spatial mode is divided into $\Gamma^{\prime}(e, k)$ and $\left|\Gamma^{\prime}(e, k)\right|=|\Gamma(e, k)|$. Moreover, the free segment $\gamma_{e k}$ is reduced to the size of $\gamma_{e k}^{\prime}$. Now, let us assume that we select other SCh $s c h^{\prime \prime}$ within the segment $\gamma_{e k}$ with a starting slot index higher by $i$ than the starting slot of SCh $s c h^{\prime}$ and which is not right-bordering. As the new SCh is selected within the same free segment, all slots between $s c h_{\text {start }}^{\prime}$ and $s c h_{\text {end }}^{\prime \prime}$ must not be occupied in spatial mode with $\Gamma(e, k)$ free segments. In consequence, the number of free segments increases, i.e., $\left|\Gamma^{\prime \prime}(e, k)\right|=\left|\Gamma^{\prime}(e, k)\right|+1$, and the free segment $\gamma_{e k}^{\prime}$ is divided into two smaller segments, $\gamma_{e k}^{\prime \prime \alpha}$ and $\gamma_{e k}^{\prime \prime \beta}$, located to the left and right side of $s s h^{\prime \prime}$, respectively. The sizes of newly created free segments are equal to $\left|\gamma_{e k}^{\prime \prime \alpha}\right|=i$ and $\left|\gamma_{e k}^{\prime \prime \beta}\right|=\left|\gamma_{e k}^{\prime}\right|-i$. Let us assume that the allocation of $s c h^{\prime}$ results in better spatial mode fragmentation $F_{S m}^{R M S F^{\prime}}(e, k)$ than allocation of any other $s c h^{\prime \prime}$ (such fragmentation is denoted as $F_{s m}^{R M S F^{\prime \prime}}(e, k)$ ). Thus,

$$
\begin{aligned}
F_{s m}^{R M S F^{\prime}}(e, k) & \leq F_{s m}^{R M S F^{\prime \prime}}(e, k) \Rightarrow \\
& \Rightarrow \frac{s_{e k}^{\prime m a x} \cdot\left|\Gamma^{\prime}(e, k)\right|}{\sqrt{\frac{\sum_{\gamma_{e k} \in \Gamma^{\prime}(e, k)}\left|\gamma_{e k}\right|^{2}}{\left|\Gamma^{\prime}(e, k)\right|}}} \leq \frac{s_{e k}^{\prime \prime m a x} \cdot\left|\Gamma^{\prime \prime}(e, k)\right|}{\sqrt{\frac{\sum_{\gamma_{e k} \in \Gamma^{\prime \prime}(e, k)}^{\left|\Gamma^{\prime \prime}(e, k)\right|} \gamma_{e k}}{2}}}
\end{aligned}
$$

Which is equivalent to:

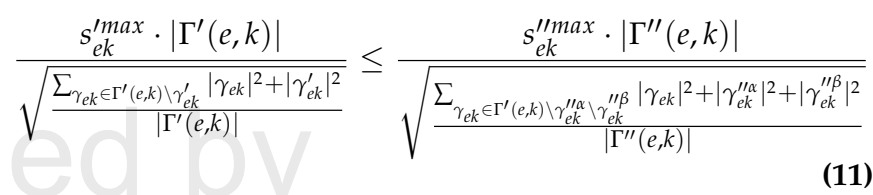

It can be observed that $s_{e k}^{\prime \prime m a x} \geq s_{e k}^{\prime \max },\left|\Gamma^{\prime \prime}(e, k)\right|>\left|\Gamma^{\prime}(e, k)\right|$, and $\left|\gamma_{e k}^{\prime \prime \alpha}\right|^{2}+\left|\gamma_{e k}^{\prime \prime}\right|^{2} \leq\left|\gamma_{e k}^{\prime}\right|^{2}$, and as the consequence, the above equation is true. Therefore, the fragmentation metric yielded by using any non-bordering $\mathrm{SCh}$ is worse than the metric when the nearest left-B-SCh is considered, which ends the proof.

Lemma 4.2. To find SCh of size $n_{d p}$ which minimizes fragmentation metric ABP on given spatial mode $k \in K$ of link $e \in E$, it is sufficient to check all (and only) bordering SChs of that size and select the one which minimizes the fragmentation metric the most.

Proof. The first considerations are analogous to the proof of Lemma 4.1. In consequence, it is sufficient to show that

$$
F_{s m}^{A B P^{\prime}}(e, k) \leq F_{s m}^{A B P^{\prime \prime}}(e, k)
$$

and as on both sides the denominators are equal (because the total number of free slots is the same after assignment in either case), it corresponds to showing that

$$
\forall_{g \in G}\left\lfloor\gamma_{e k}^{\prime} / g\right\rfloor \geq\left\lfloor\gamma_{e k}^{\prime \prime \alpha} / g\right\rfloor+\left\lfloor\gamma_{e k}^{\prime \prime \beta} / g\right\rfloor
$$

which is obviously true and ends the proof.

\section{B. Impact of Bordering SChs on Link Fragmentation}

The lemmas above may be easily extended to show that checking all B-SChs of each spatial mode in the fiber allows finding a SCh that minimizes fragmentation of the entire fiber (as defined in Eq. (2)).

Lemma 4.3. Assume that finding B-SChs for given spatial mode $k$ assures finding a SCh whose allocation yields minimal spatial mode fragmentation. To find SCh which minimizes fiber fragmentation, it is sufficient to check all (and only) B-SChs for each spatial mode of that fiber, and select the one for which the difference between the corresponding spatial mode fragmentation after and before SCh allocation is the lowest.

Proof. Let us assume that for each spatial mode $k \in K$ we can find a B-SCh whose allocation results in the lowest fragmentation on that spatial mode. The fragmentation of spatial mode $k$ of edge $e$ before that B-SCh allocation is denoted as $F_{s m}^{m}(e, k)$, while after as $F_{s m}^{m}{ }^{\prime}(e, k)$. Let us assume that the selection of B-SCh on spatial mode $k^{*}$ results in the lowest fragmentation. Thus, the 
fiber fragmentation after allocation of SCh on spatial mode $k^{*}$ is equal to:

$$
F_{\text {link }}^{m}(e)=\frac{\sum_{k \in K} F_{s m}^{m}(e, k)-F_{s m}^{m}\left(e, k^{*}\right)+F_{s m}^{m}{ }^{\prime}\left(e, k^{*}\right)}{|K|}
$$

Thus,

$$
k^{*}=\underset{k \in K}{\arg \min }\left(F_{s m}^{m}{ }^{\prime}(e, k)-F_{s m}^{m}(e, k)\right)
$$

which ends the proof.

\section{Representation of SChs in a Path}

The creation of a SCh over a path depends on the applied scenario. In the case of SCC, SChs are realized on the same spatial mode $k \in K$ of each link $e \in E(p)$ in the path $p \in P$. Thus, the SCh over a path with SCC may be uniquely described as a tuple $\left(\left(s c h_{\text {start }}, s c h_{\text {end }}\right), k\right)$. In the case of SCC relaxed, it would be required to store information on which spatial mode of which link the SCh is realized. However, we assume that SCh of a given starting and ending index on a given link is realized on the first spatial mode, on which a given channel is free. Thus, for our proposed algorithm, it is sufficient only to represent a super-channel as a tuple $\left(s c h_{\text {start }}, s c h_{\text {end }}\right)$.

\section{FRAGMENTATION-AWARE ALGORITHM}

In this section, we present our proposed Fragmentation-Aware algorithm with bordering super-channels (FA-BSC) aimed at minimizing the blocking probability by minimizing overall network fragmentation.

\section{A. Finding Bordering Super-Channels}

To this end, an algorithm for finding B-SChs is required. In Algorithm 1, helper functions are presented: i) function FindNextAllocated finds the index of the lowest slot in slot array $s$ that is allocated and it is higher or equal to the provided index $i$. If the end of slots array is reached, -1 is returned; ii) function FindNextFree finds an index of the lowest slot in slots array $s$ that is free and it is higher or equal to the provided index $i$. If the end of slots array is reached, -1 is returned; iii) function FindNextFreeSize finds the lowest contiguous subset of free slots in slots array $s$ that is at least of size size and the starting slot is higher than the provided index $i$. If such a subset is found, the starting index of that subset is returned, -1 otherwise.

Algorithm 2 presents pseudocode of Find Bordering SuperChannels (FBS) method which finds a set of B-SChs of size size in slots array s. In lines 2-3 variables are initialized, i.e., set of found B-SChs $s c h_{b}$ as the best one to realize request and currently considered minimum SCh starting index $i$. Next, in a loop, consecutive B-SChs are found (line 4) until the minimum index is higher than the size of slots array minus the size of sought B-SCh size. In line 5, a starting slot of the next free segment of at least size size is obtained as left. If it is not possible to find such a free segment, set of already found B-SCh $s c h_{b}$ is returned. Next, the candidate left-bordering SCh sch is created (line 9) and the minimum index is moved one slot higher than the ending slot index of that B-SCh. Then, given left-bordering $\mathrm{SCh}$ is added to the currently found B-SChs $\operatorname{sch}_{b}$ (line 11). Next, it is checked, whether the considered $\mathrm{SCh}$ is also right-bordering. To do so, in line 12, the adjacent slot index to the left B-SCh is investigated whether it is free or not. If it is not, the SCh is also right-bordering and the while loop repeats seeking for the next
Algorithm 1. Helper functions

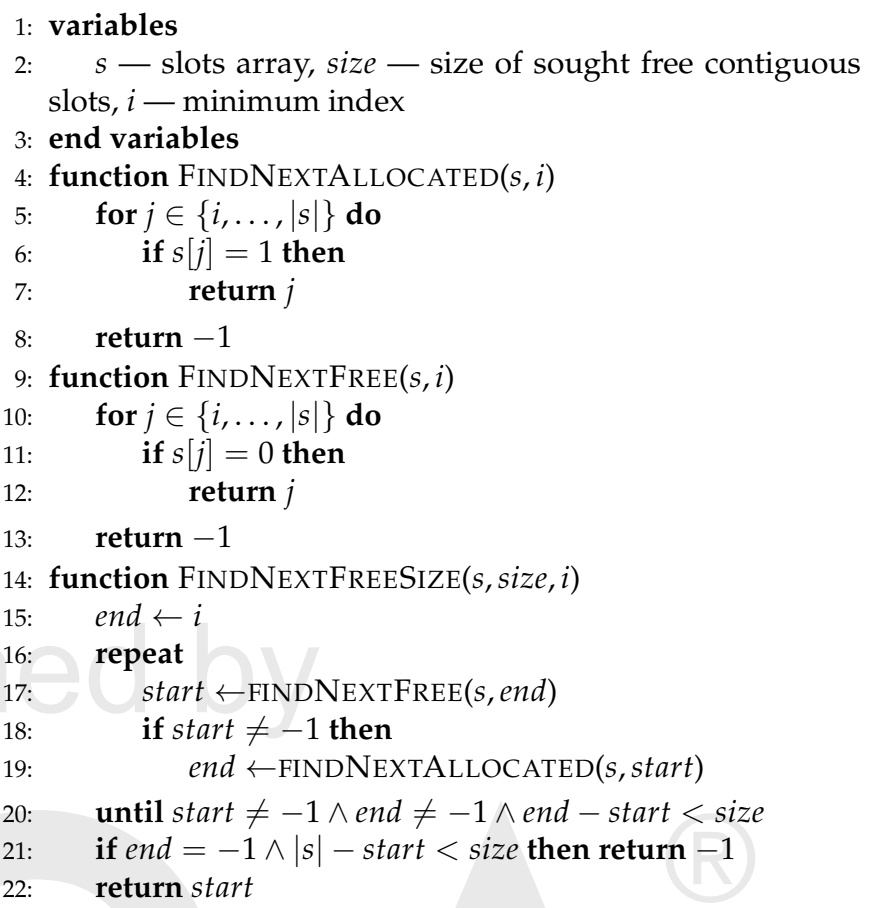

left B-SCh. Otherwise, the right B-SCh is obtained by looking for next allocated slot index (line 13) stored in variable right. If right is not -1 , it indicates that the right-bordering SCh may be created as (right - size, right - 1) and added to the currently found B-SCh $\operatorname{sch}_{b}$ (lines 14-17). Otherwise, the end of fiber is reached, and the B-SCh should be created adjacent to the end (line 18) and then added to the currently found ones. In both cases, the minimum slot index $i$ is updated. When the whole fiber is investigated, the while loop exits and the set of B-SCh $s c h_{b}$ is returned.

Algorithm 2. Find Bordering Super-Channels

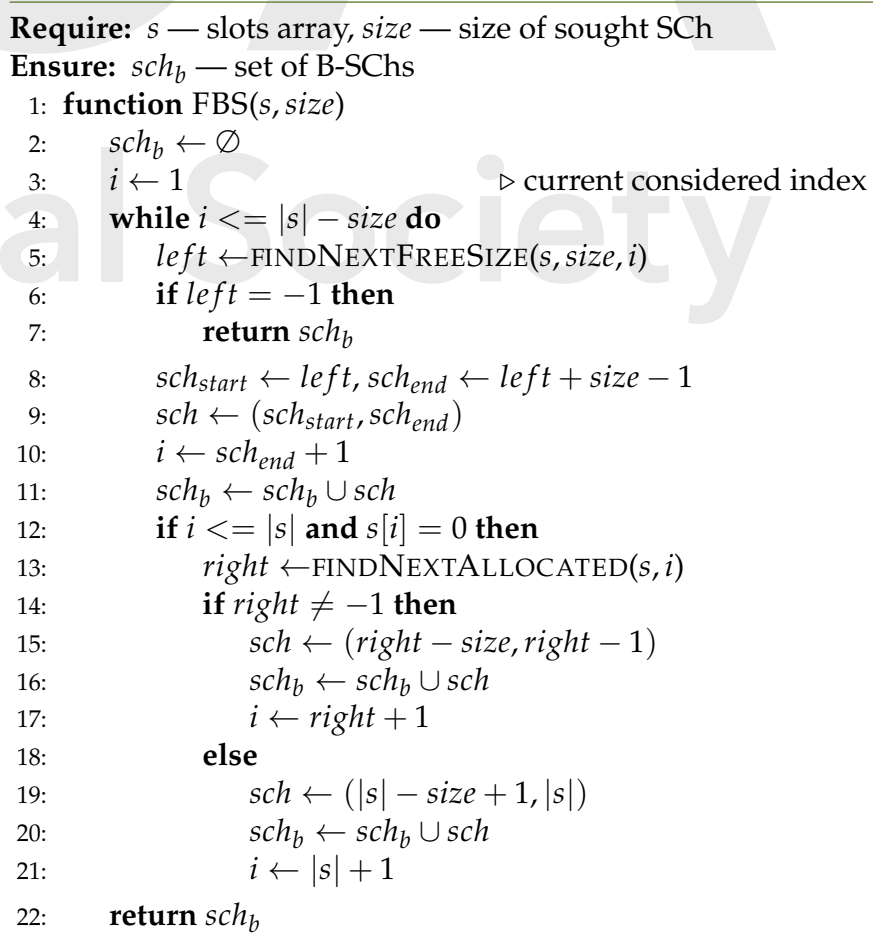


Note that Algorithm 2 as presented always looks for a superchannel of size slots, where size includes one slot for the guardband. If the guardband is treated as occurring at the right of the super-channel, then at the very high end of the spectrum, a guardband is not needed because there can be no super-channel to the right of it. However, this can be compensated by the assumption that at the end of each fiber there is one additional extra slot (which is physically not available).

\section{B. Create Fiber Representing Path with SCC}

We propose a procedure that finds candidate SChs based on the B-SChs on a given path $p \in P$ assuming spatial continuity constraint. To this end, a fiber representing path (FREP) when SCC is assumed $f_{p}^{O R, S C C}$ is created using OR operators. Let us assume that path $p \in P$ contains $F(p)=\left\{f_{1}, f_{2}, \ldots, f_{p}\right\}$ fibers We assume that a super-channel is carried on a single fiber on any link of the path; thus, a path with $\mathrm{p}$ links traverses $\mathrm{p}$ fibers. Each fiber contains $K$ spatial modes. Let $f_{i, k}$ denote a spatial mode $k \in K$ of fiber $f_{i}$. Analogously, let $f_{p, k}^{O R, S C C}$ denote a spatial mode $k$ in FREP. The proposed method applies logical OR function between corresponding spatial modes of each fiber in the path. Each spatial mode of FREP is defined as:

$$
\forall_{k \in K} f_{p, k}^{O R, S C C}=f_{1, k} \vee f_{2, k} \vee \ldots \vee f_{p, k}
$$

where the logical OR function between two spatial modes, e.g., $k_{\alpha}$ and $k_{\beta}$ results in the creation of new spatial mode $k^{\prime}$ for which

$$
\forall_{i \in 1, \ldots,|S|} s_{k^{\prime}}[i]=s_{k_{\alpha}}[i] \vee s_{k_{\beta}}[i]
$$

Figure 2 depicts the process of creating FREP with SCC. Gray squares denote allocated slots, while white ones stand for free ones. For example, fiber $f_{1}$ contains 4 occupied slots on spatial mode $k_{1}$ with indices $\left\{s_{1}, s_{2}, s_{7}, s_{8}\right\}$. Fiber $f_{2}$ on spatial mode $k_{1}$ has 8 allocated slots from slot $s_{1}$ to $s_{8}$. Fiber $f_{p}$ on spatial mode $k_{1}$ has five free slots, $s_{10}$ and from $s_{1}$ to $s_{4}$. After applying logical OR operator between spatial modes $k_{1}$ of each fiber, the spatial mode $k_{1}$ of FREP is created which has 9 allocated slots from $s_{1}$ to s9.

Let $B S C h_{S C C}\left(p, n_{d p}\right)$ denote a set of candidate B-SChs for path $p \in P$ of size $n_{d p}$ when SCC is considered which is defined as

$$
\begin{array}{r}
B S C h_{S C C}\left(p, n_{d p}\right)=B S C h_{S C C}\left(f_{p}^{O R, S C C}, n_{d p}\right)= \\
=\bigcup_{k \in K}\left\{\left(\left(s c h_{\text {start }}, s c h_{\text {end }}\right), k\right):\right. \\
\left.:\left(s c h_{\text {start }}, s c h_{\text {end }}\right) \in B S C h\left(f_{p}^{O R, S C C}, k, n_{d p}\right)\right\}
\end{array}
$$

Considering output FREP from Fig. 2 and size of SChs of $n_{d p}=2$ slots, set BSCh $h_{S C C}$ would contain 4 SChs, i.e. $\left(s_{1}, s_{2}\right)$ and $\left(s_{3}, s_{4}\right)$ on $k_{3}$, as well as $\left(s_{4}, s_{5}\right)$ and $\left(s_{5}, s_{6}\right)$ on $k_{2}$.

\section{Create Fiber Representing Path with SCC Relaxed}

The process of creating FREP when SCC is relaxed on path $p \in P$ is slightly modified when compared to a case with SCC. As a result, a $f_{p}^{O R, n o S C C}$ (fiber representing path using OR operators when SCC is relaxed) is created. First, for each fiber except the first one in the path, logical AND operator is applied between all spatial modes in that fiber creating A-Fibers (fibers using AND operator). The A-Fiber $f_{i}^{A N D}$ for fiber $f_{i}$ is defined as:

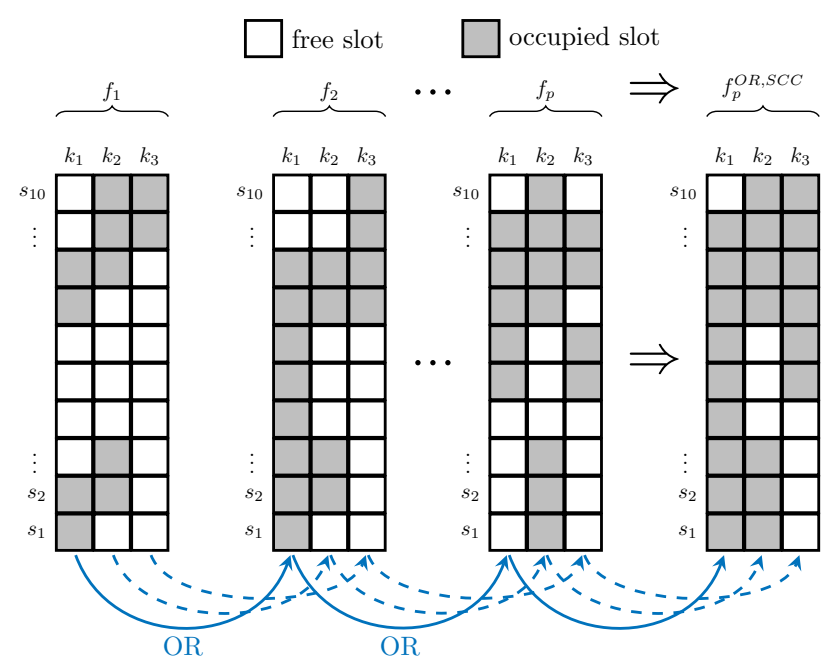

Fig. 2. Creating fiber representing path with spatial continuity constraint

$$
f_{i}^{A N D}=f_{i, k_{1}} \wedge f_{i, k_{2}}, \ldots, f_{i, k_{|K|}}
$$

where the logical AND function between two spatial modes, e.g., $k_{\alpha}$ and $k_{\beta}$ results in the creation of new spatial mode $k^{\prime}$ for which

$$
\forall_{i \in 1, \ldots,|S|} s_{k^{\prime}}[i]=s_{k_{\alpha}}[i] \wedge s_{k_{\beta}}[i]
$$

Next, to obtain the $f_{p}^{O R, n o S C C}$, the logical OR function is applied between each spatial mode of the first fiber and all A-Fibers created from the remaining fibers of the path

$$
\forall_{k \in K} f_{p, k}^{O R, n o S C C}=f_{1, k} \vee f_{2}^{A N D} \vee f_{3}^{A N D} \vee \ldots \vee f_{p}^{A N D}
$$

Figure 3 depicts the process of creating FREP with SCC relaxed assuming the same occupied slots in each fiber as in Fig. 2. As the first step, the A-Fiber is created for all fibers except the $f_{1}$. For example, $f_{2}^{A N D}$ A-Fiber results from applying logical AND operator for all spatial modes in fiber $f_{2}$. The $f_{2}^{A N D}$ fiber has allocated two slots $s_{7}$ and $s_{8}$. Similarly, fiber $f_{p}$ is transformed into fiber $f_{p}^{A N D}$ which has two allocated slots $s_{8}$ and $s_{9}$. Next, the $f_{p}^{O R, n o S C C}$ fiber is created by applying logical OR operators. Spatial mode $k_{1}$ of FREP is created by applying OR operator with spatial mode $k_{1}$ of fiber $f_{1}$ and remaining A-Fibers $-f_{2}^{A N D}$ through $f_{p}^{A N D}$. As the spatial continuity relaxation allows for higher flexibility, the resulting FREP has fewer occupied slots than the corresponding one when SCC is assumed (see Fig. 2).

Analogously to Eq. (18), the set of candidate B-SChs $B S C h_{n o S C C}\left(p, n_{d p}\right)$ for path $p \in P$ of size $n_{d p}$ when SCC is relaxed is defined as

$$
\begin{array}{r}
B S C h_{n o S C C}\left(p, n_{d p}\right)= \\
=\bigcup_{k \in K} \operatorname{BSCh} h_{n o S C C}\left(f_{p}^{O R, n o S C C}, n_{d p}\right)= \\
\{s c h=(i, j):(i, j) \text { is realized on } k \wedge \\
\left.\exists_{k^{\prime} \in K}\left(k^{\prime}<k \wedge(i, j) \text { is realized on } k^{\prime}\right)\right\}
\end{array}
$$

The difference is that if the SCh containing the same starting and ending slot is found on more than one spatial mode, it is 
removed from spatial modes with higher indices. The reasoning is that the SCC is relaxed, thus, this SCh on the particular core will be covered by applying the SCh finding method.

Considering output FREP from Fig. 3 and size of SChs of $n_{d p}=2$ slots, set $B S C h_{n o S C C}$ would contain 4 SChs, namely, $\left(s_{3}, s_{4}\right),\left(s_{5}, s_{6}\right)$ on $k_{1},\left(s_{4}, s_{5}\right)$ on $k_{2}$, and $\left(s_{1}, s_{2}\right)$ on spatial mode $k_{3}$. Note, $\left(s_{5}, s_{6}\right)$ on spatial modes $k_{2}$ and $k_{3}$ was removed from the set (as they are the duplicates on other spatial modes).

The proposed FREP creating method due to the logical AND function applied on some fibers may result in finding some BSChs in the set $B S C h_{n o S C C}$ that are not feasible, i.e., they cannot be realized on a single spatial mode of a particular fiber and would require splitting among several spatial modes. (The example of Fig. 3 does not include any infeasible B-SChs. However, by looking at $f_{p}$, one can see how an infeasible B-SCh could potentially be produced. After ANDing the 3 spatial modes of $f_{p}$, slots 6 and 7 are both 'free'. However, if a super-channel of size 2 slots were assigned to slots 6 and 7 on $f_{p}$, it would require splitting the super-channel over $k_{2}$ and $k_{3}$, which we assume is not permitted.)

Therefore, post-processing is applied to remove unfeasible SChs from the set of candidate ones, and the algorithm considers only feasible ones that do not require splitting over several spatial modes. Alternatively, the pre-processing may be applied to each fiber marking the free slots that do not have enough neighbors to support the whole SCh as "occupied" ones (in this case, the $B S C h_{n o S C C}$ set would contain only feasible SChs). As another alternative, a fixing routine may be applied which rearranges the connections in a single fiber according to [28] (it is always possible to re-arrange connections in fibers along the path in a way that there exists as wide free contiguous set of slots on a single spatial mode as presented in the $f_{p}^{O R, n o S C C}$ fiber).

Moreover, when SCC is relaxed, any spatial mode of given input fiber may be freely directed to any spatial mode of given output fiber. Thus, SCh of particular starting and ending slot index $(i, j)$, may be realized on more than one spatial mode of a certain fiber. In such a case, the general idea is to select the spatial mode with lower index (space-first approach). Note that this approach does not guarantee minimal fragmentation, but it allows to reduce additional computational complexity required to check all spatial modes selection possibilities. Checking all spatial modes combinations has $O\left(|K|^{|E(P)|}\right)$ complexity where $|K|$ denotes number of spatial modes and $|E(P)|$ denotes number of edges in the path. Contrarily, the space-first approach requires checking first free spatial mode on each fiber, which has complexity $O(|K| \cdot|E(p)|)$.

It is also worth mentioning, that obtained sets $B S C h_{S C C}\left(p, n_{d p}\right)$ and $B S C h_{n o S C C}\left(p, n_{d p}\right)$ of candidate SChs of size $n_{d p}$ on routing path $p$ (based on FREPs $f_{p}^{O R, S C C}$ and $f_{p}^{O R, n o S C C}$ respectively) are not necessarily bordering in the sense that they are bordering on all fiber links along the routing path. We only know that they are bordering on at least one of the fibers, but we expect that this will still reduce fragmentation.

\section{Fragmentation-Aware with Bordering SChs Algorithm}

To minimize overall network fragmentation, we designed a Fragmentation-Aware algorithm which uses Bordering SuperChannels (FA-BSC), presented in Algorithm 3. In line 2, network fragmentation metric $F_{n e t}^{m *}$ is initialized to 0 and the algorithm iterates through all pending requests $D$ (line 3 ). Next, for each demand $d \in D$, two variables are initialized, $s c h^{*}$ and $F_{n e t}^{m * \prime}$

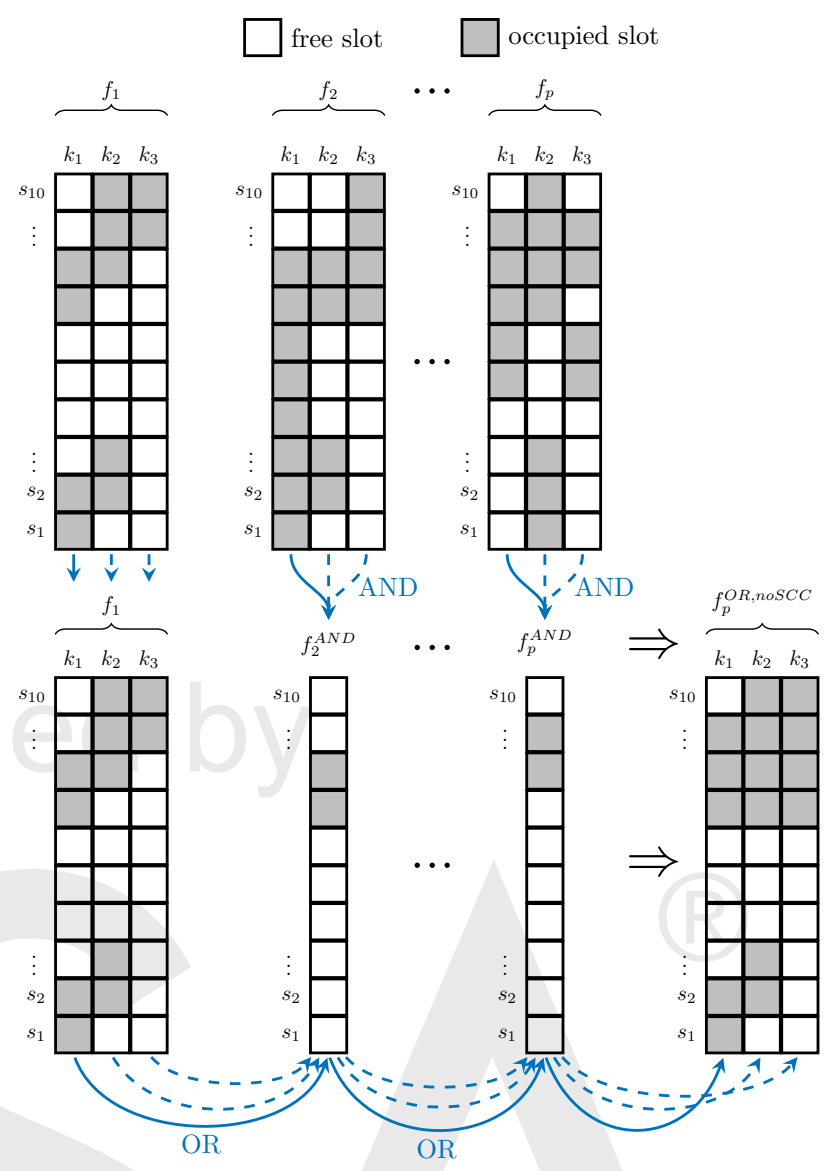

Fig. 3. Creating fiber representing path with spatial continuity constraint relaxed.

which correspond to the best SCh for a given demand and fragmentation metric after allocation of that demand on a given super-channel (lines 4-5). In line 6 the algorithm iterates through each candidate path for the considered request in order to find the best SCh for allocation (generation of the candidate paths is discussed later). In next steps, the execution depends on whether SCC is assumed or not (line 7). If the SCC is considered, the set of B-SChs BSCh is created based on the FREP $f_{p}^{O R, S C C}$ that assumes SCC (lines 8-9). However, if the SCC is relaxed, the set of B-SChs $\mathrm{BSCh}$ is created based on corresponding FREP $f_{p}^{O R, n o S C C}$ (lines 11-12). Next, if it was possible to find any B$\mathrm{SCh}$, the algorithm iterates through this set in line 14. If the SCC is considered, the SCh sch is allocated on corresponding spatial mode on all links in the path (line 16). Otherwise, the SCC is relaxed, and SCh sch is allocated on channel using spatial modes with the lowest index on which that channel is free, on each link in the path (line 18). Next, in line 19 the network fragmentation $F_{n e t}^{m} '$ is calculated after allocation of SCh sch. If allocation of considered SCh sch yields lower fragmentation than the currently best fragmentation that has been found (line 20), the SCh sch and fragmentation metric $F_{n e t}^{m}$ ' are stored as the current best ones, i.e., $s c h^{*}$ and $F_{n e t}^{m * \prime}$, respectively (lines 21-22). Next, it is required to remove allocated SCh sch in order to investigate other SChs. The steps are repeated until all B-SCh for all candidate paths for demand $d$ are evaluated. After that, if for particular request $d$ it was possible to find the best SCh $s c{ }^{*}$ (line 24), this request is realized using that $\mathrm{SCh}$ and total network fragmentation is updated (lines 25-26). Otherwise, if the request cannot be allocated 
on any of the candidate paths, the request is blocked (line 28). Finally, these steps are repeated for all pending dynamic requests.

Algorithm 3. Fragmentation-Aware with Bordering SuperChannels (FA-BSC) Algorithm

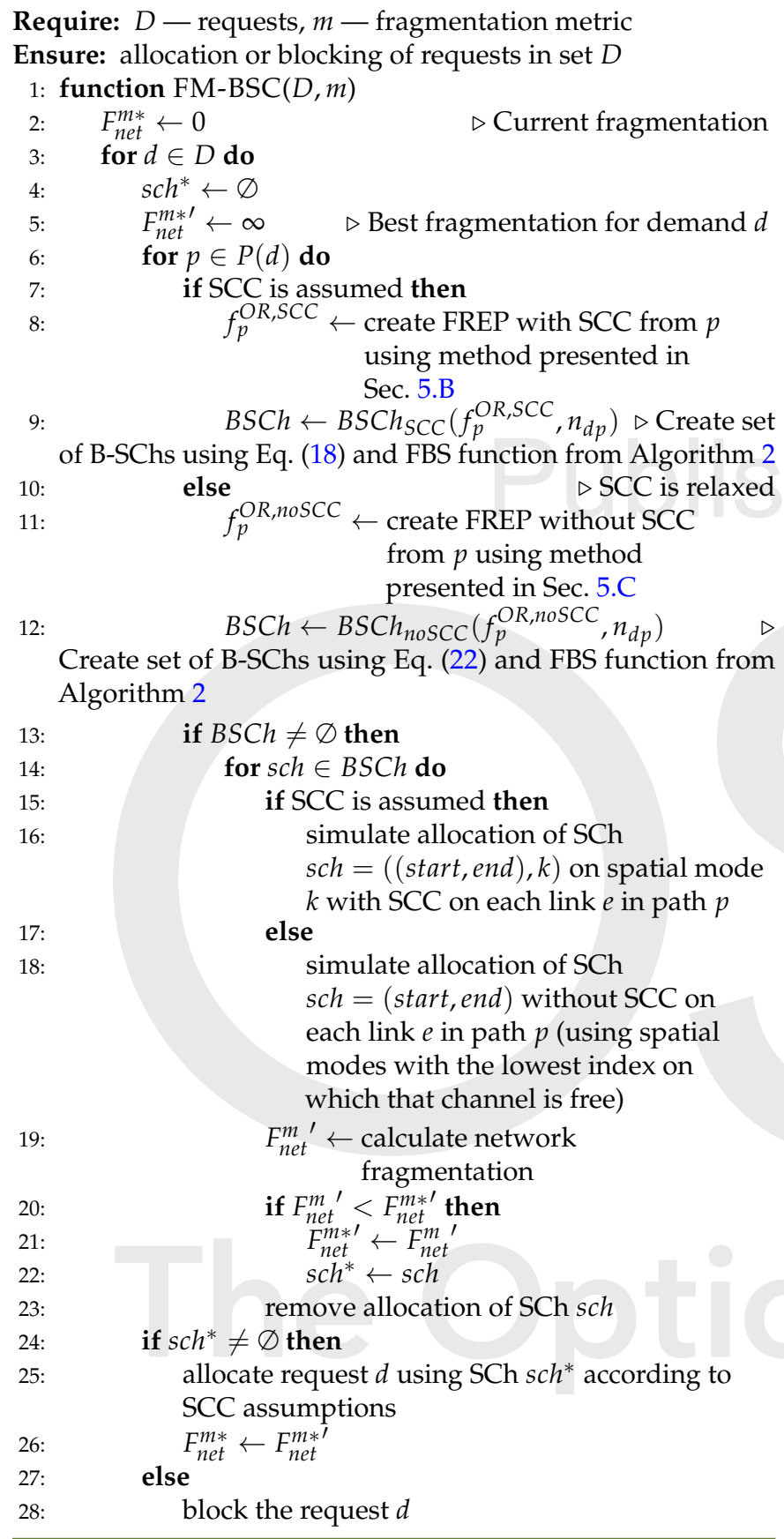

\section{E. Reference Algorithms}

As a baseline algorithm, we apply the Fragmentation Aware $k$ Shortest Path (FA-kSP) algorithm [11]. FA-kSP, for each request, considers $k$-shortest candidate paths and for each path, it selects the SCh with the lowest ending slot index. If more than two super-channels have the same starting slot index on a given path, the space-first policy is used. Next, the candidate set of SChs is created based on the SChs found for each path. Further, these candidate SChs are compared according to provided fragmentation metric (same as in this paper) and the one which provides the lowest network fragmentation is selected.

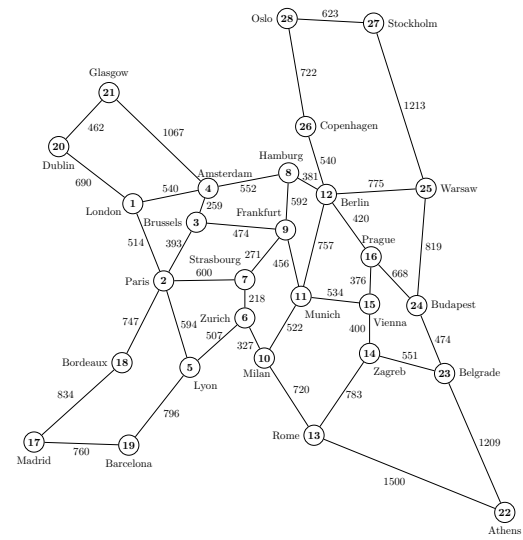

Fig. 4. Euro28 network topology.

Also, we compare the results to the $k$-shortest paths first fit (FF) algorithm, which allocates each request on the first available SCh found after investigating a given set of $k$-shortest candidate routing paths (i.e., on given path, the algorithm finds SCh with the lowest slice index, and in case of a draw, with the lowest spatial mode index on each fiber).

Moreover, to show the advantage of selecting B-SCh over other SChs, we compare FA-BSC to a simplified version of FABSC called Fragmentation-Aware with Multiple Super-Channels (FA-MSC) algorithm. FA-MSC considers exactly the same number of candidate SChs as FA-BSC (i.e., $\left|B S C h_{S C C}\right|$ or $\left|B S C h_{n o S C C}\right|$, depending on the scenario), which assures fair comparison, but it does not apply the additional logic of selecting bordering SChs. In more detail, for each request, it investigates k-shortest paths, and for each path, it calculates how many candidate SChs would be selected using the FA-BSC heuristic. Next, it selects the same number of candidate SChs for a given path. The first selected $\mathrm{SCh}$ is the one with the lowest ending slot index (as in FA-kSP) and the remaining ones are selected randomly. Finally, it compares created candidate set of SChs along all $k$-shortest paths using the provided fragmentation metric as in FA-BSC.

Note that in our descriptions of the algorithms, we assume that there is a set of $k$-shortest candidate paths generated with Yen's algorithm, however, any strategy for generating candidate paths can be used.

\section{NUMERICAL EXPERIMENTS}

We run numerical experiments to evaluate the performance of the proposed fragmentation-aware algorithm under dynamic traffic in SS-FON. We consider a European network topology with 28 nodes, 82 links, and an average link length of $625 \mathrm{~km}$ (Fig. 4). Each link comprises 12 spatial modes, and each spatial mode provides $4 \mathrm{THz}$ of bandwidth. The network operates within a flexible grid of 320 slots, each $12.5 \mathrm{GHz}$ width.

Dynamic traffic requests follow a Poisson process with an average arrival rate of $\lambda=10$ requests per unit time. The lifetime of each request follows a negative exponential distribution with an average of $1 / \mu$. Thus, the traffic load equals $\lambda / \mu$ normalized traffic units (NTUs). Each traffic request has bit-rate selected randomly within $50 \mathrm{Gbps}$ to $1 \mathrm{Tbps}$ range, with $50 \mathrm{Gbps}$ granularity. Therefore, traffic of 100 NTUs refers to around $52.5 \mathrm{~Tb} / \mathrm{s}$ of overall traffic load. We study two types of request distributions: i) traffic $A$ - the source and destination nodes are selected with uniform distribution; ii) traffic $B$ - the source and destination nodes are selected inversely proportionally to the distance in kilometers between them (i.e., closer node pairs are selected 


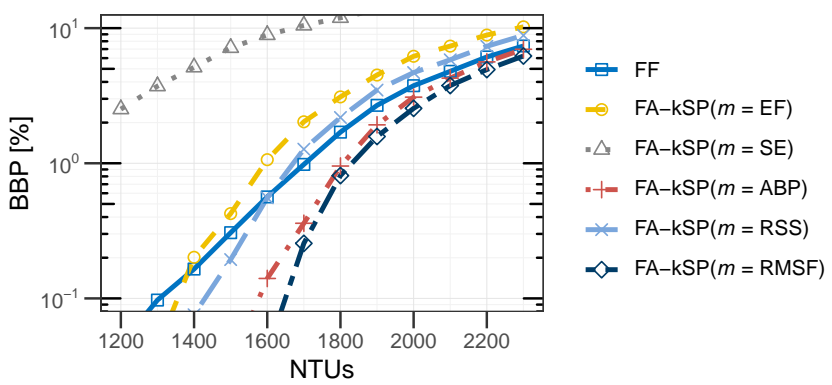

(a) No spatial continuity constraint

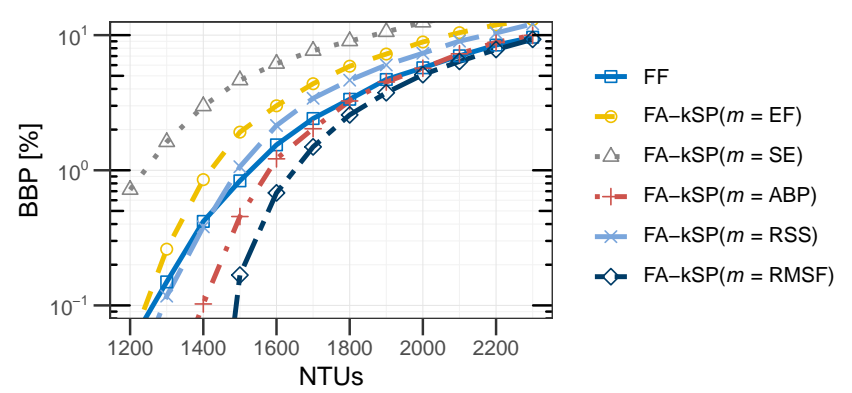

(b) With spatial continuity constraint.

Fig. 5. BBP of FA-kSP algorithm with various used metrics as a function of NTUs, traffic A.

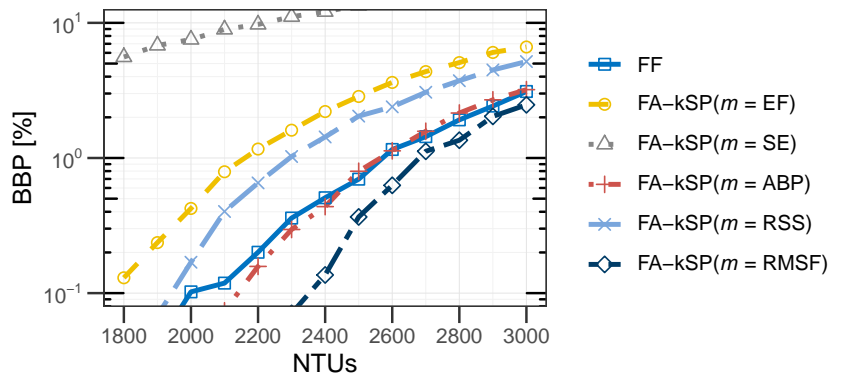

(a) No spatial continuity constraint

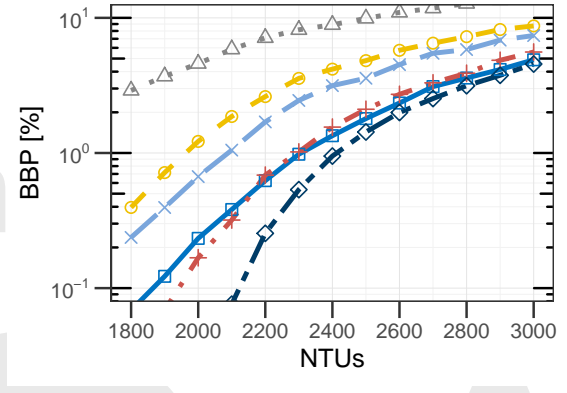

(b) With spatial continuity constraint.

Fig. 6. BBP of FA-kSP algorithm with various used metrics as a function of NTUs, traffic B.

with higher probability). For each analyzed value of traffic load, one set of 36000 requests is offered to the network. To enable the network reaching a steady state, initially additional 4000 requests are processed. For each request, the number of candidate paths for each algorithm is 10 , representing the 10 shortest paths. Moreover, two scenarios are considered, with spatial continuity (SCC) and with spatial continuity relaxed (no SCC).

The performance metric is bandwidth blocking probability (BBP), defined as the sum of bit-rates of rejected requests, over the sum of bit-rates of all requests offered to the network. Additionally, we use the accepted traffic metric for a $1 \%$ threshold of BBP. Accepted traffic corresponds to the value of traffic load in NTUs, for which BBP is approximately $1 \%$ on average.

The first experiment compares FA-kSP algorithm employing various fragmentation metrics, with the first-fit $k$-shortest path (FF) algorithm. Figure 5 presents BBP as a function of NTUs for various fragmentation metrics used in FA-kSP algorithm for traffic A. Note, the name FA-kSP $(m=$ metric $)$ denotes FA-kSP algorithm utilizing fragmentation metric "metric". Firstly, the RMSF metric allows FA-kSP algorithm to achieve the lowest BBP for all NTUs, regardless of whether spatial continuity is present or not. ABP metric achieves second-best results. For example, for 1800 NTUs and SCC relaxed, RMSF metric decreases the BBP by $15.1 \%$ and $52.3 \%$ with respect to the second-best strategy, ABP, and the reference one, FF, respectively. For traffic A, RMSF allows approximately accommodating $2.6 \%$ and $7.4 \%$ more NTUs than ABP and FF, achieving BBP below $1 \%$. Moreover, the relaxation of SCC allows accepting approximately $12.6 \%$ more NTUs for a given metric (i.e., Fig. 5a vs. Fig. 5b).

In Fig. 6 corresponding results are presented for traffic $B$. Similar to traffic A, RMSF provides lowest BBP, e.g., for 2600 NTUs, BBP for RMSF is $45.7 \%$ and $44.4 \%$ lower than FF and ABP, respectively when SCC is relaxed. On average, relaxing SCC allows accommodating $10.8 \%$ more NTUs when compared to the case with SCC for $1 \%$ of BBP. Finally, due to the characteristic of traffic, it is possible to provide $43.6 \%$ more NTUs comparing with uniform distribution (i.e., Fig. 5 vs. Fig. 6).

In the second part of the experiments, we focus on the improvements achieved by searching only bordering superchannels. To this end, we selected the two best fragmentation metrics, RMSF and ABP, and verified their performance using FA-BSC algorithm. As the reference, FA-kSP algorithm and $k$ shortest path First Fit (FF) algorithm is used [11]. To show the advantage of selecting B-SChs, the FA-BSC is also compared to FA-MSC, which selects the same number of candidate SChs for comparison without a specified strategy.

Figure 7 shows BBP as a function of NTUs for various algorithms using the RMSF metric. The BSC algorithm is shown with solid lines. As can be observed, for SCC relaxed (left), investigation of bordering SChs in FA-BSC improves BBP on average by $20.4 \%$ and $10.2 \%$ compared to FA-kSP and FA-MSC, respectively, for $\mathrm{BBP}$ above $0.05 \%$ (i.e., when the blocking for considered traffic for both algorithms is above $0.05 \%$ ). For example, for 1800 NTUs, FA-BSC yields $0.57 \%$ BBP, while for FA-kSP and FA-MSC it is $0.81 \%$ and $0.67 \%$, respectively. For SCC (right), the improvement equals to $9.5 \%$ and $13.6 \%$ compared to FA-kSP and FA-MSC, respectively. Furthermore, FA-BSC algorithm allows providing approximately $1.7 \%$ and $1.2 \%$ more NTUs achieving 1\% BBP threshold, when compared to FA-kSP and FA-MSC algorithms (averaged over both traffic types and both spatial continuity assumptions). Figure 8 presents BBP as a function of NTUs for algorithms using ABP metric, for Traffic A and SCC relaxed. Bordering SChs in FA-BSC improve BBP on average by $23.1 \%$ and $8.2 \%$ compared to FA-kSP and MSC, respectively, for BBP above $0.05 \%$. Figure 9 shows BBP as a function of NTUs for algorithms using RMSF metric, for Traffic B and SCC relaxed. As can be observed, the trend for other traffic profile is similar, i.e., BSC algorithm improves BBP on average by $22.9 \%$ and $20.7 \%$ compared to FA-kSP and MSC, respectively, for BBP above $0.05 \%$. 


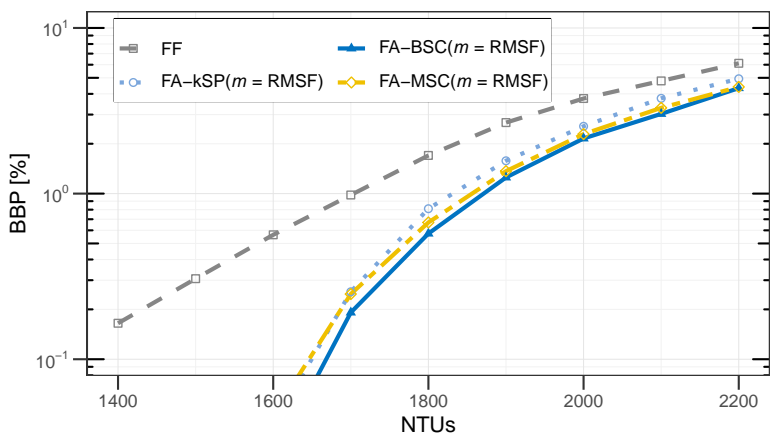

(a) No spatial continuity constraint.

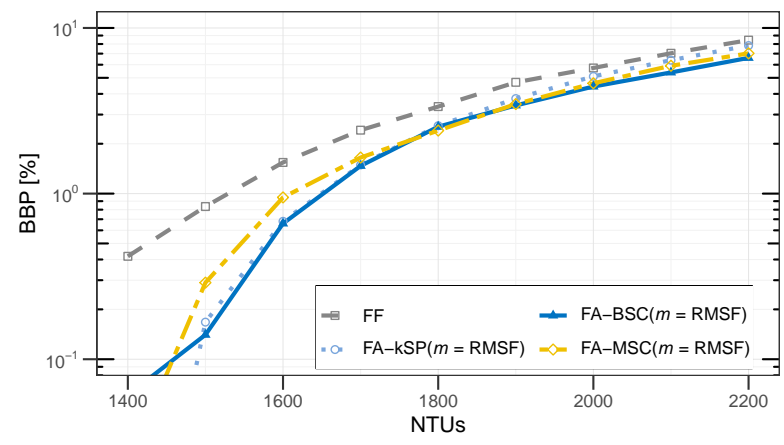

(b) With spatial continuity constraint.

Fig. 7. BBP of FA-BSC, FA-kSP, FA-MSC and FF as a function of NTUs for RMSF fragmentation metric, traffic A.

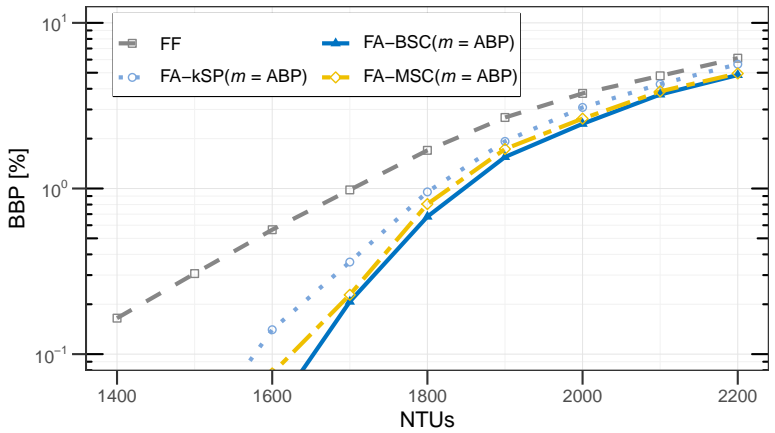

Fig. 8. BBP of FA-BSC, FA-kSP, FA-MSC and FF as a function of NTUs for ABP fragmentation metric, spatial continuity constraint relaxed, traffic A.

Table 4. Improvement using bordering super-channels over other algorithms for RMSF fragmentation metric.

\begin{tabular}{|c|c|c|c|c|c|}
\hline & \multicolumn{2}{|c|}{$\mathrm{BBP}$} & \multicolumn{2}{|c|}{$\begin{array}{c}\text { Accepted traffic } \\
\text { for } 1 \% \mathrm{BBP}\end{array}$} \\
\hline & & no SCC & SCC & no SCC & SCC \\
\hline \multirow{2}{*}{ 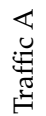 } & over FA-kSP & $20.4 \%$ & $9.5 \%$ & $2.1 \%$ & $0.2 \%$ \\
\hline & over FA-MSC & $10.2 \%$ & $13.6 \%$ & $0.8 \%$ & $2.2 \%$ \\
\hline \multirow{2}{*}{ 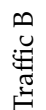 } & over FA-kSP & $22.9 \%$ & $5.8 \%$ & $3.0 \%$ & $1.5 \%$ \\
\hline & over FA-MSC & $20.7 \%$ & $20.0 \%$ & $0.2 \%$ & $1.4 \%$ \\
\hline
\end{tabular}

Table 5. Accepted traffic [NTUs] for various algorithms for RMSF fragmentation metric.

\begin{tabular}{l|ccccc}
\hline & \multicolumn{2}{c}{ Traffic A } & & \multicolumn{2}{c}{ Traffic B } \\
\cline { 2 - 3 } \cline { 6 - 6 } \cline { 5 - 6 } & no SCC & SCC & & no SCC & SCC \\
\hline FA-BSC & 1862.9 & 1642.0 & & 2754.9 & 2414.3 \\
FA-MSC & 1847.4 & 1607.4 & & 2714.7 & 2381.1 \\
FA-kSP & 1824.7 & 1639.5 & & 2675.5 & 2410.6 \\
\hline
\end{tabular}

The summary comparison between FA-BSC and reference algorithms is presented in Table 4 . As can be noticed, applying bordering SChs in fragmentation-aware algorithm improves $\mathrm{BBP}$ in all cases. Moreover, in most of the cases, the improvement is higher when SCC is not enforced. Surprisingly, when SCC is enforced, the FA-kSP algorithm provides better results

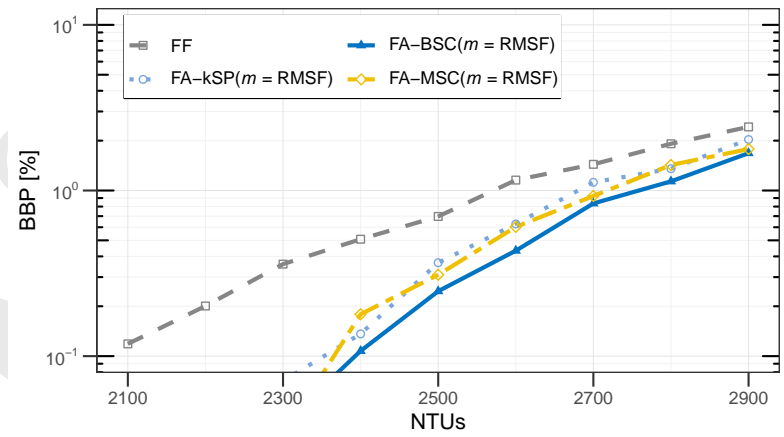

Fig. 9. BBP of FA-BSC, FA-kSP, FA-MSC and FF as a function of NTUs for RMSF fragmentation metric, spatial continuity constraint relaxed, Traffic B.

than FA-MSC. Thus, it is not always beneficial to choose multiple SChs randomly and select the one which minimizes fragmentation (recall, the FA-MSC algorithm considers also SChs with the lowest available slice as in FA-kSP). The advantage of FA-BSC comes from the applied selection strategy of SChs' subsets (i.e., a B-SChs) for further comparison. In Table 4, there is also presented improvement for accepted traffic for 1\% BBP threshold, i.e., how much more traffic may be accepted when comparing FA-BSC to other algorithms still achieving BBP below 1\%. Note, in all cases, FA-BSC algorithm allows for allocation of more traffic in the network when compared to reference algorithms.

In Table 5, accepted traffic for $1 \%$ of BBP threshold is presented for various algorithms. In all cases, FA-BSC allows accepting more traffic than reference algorithms. Also, for all algorithms, relaxation of SCC increases accepted traffic on average by $13.1 \%$. Moreover, traffic $B$ allows accepting $47.2 \%$ more traffic than traffic $\mathrm{A}$, due to the differences in their distribution.

Finally, Table 6 shows average execution time in milliseconds for a single request for various algorithms. As it may be observed, FA-BSC requires one order of magnitude higher time for single request than FF or FA-kSP. The execution time for FAMSC is slightly higher than for FA-BSC as FA-MSC is required to check how many BSCh would be selected by FA-BSC. However, if the additional penalty from higher execution time of FA-BSC is acceptable, it allows for allocating on average $1.7 \%$ more traffic assuring BBP below $1 \%$ when compared to FA-kSP.

\section{CONCLUDING REMARKS}

We have proposed several fragmentation metrics for SS-FONs as extended measurement methods of fragmentation known in EONs. Also, the novel concept of bordering super-channels 
Table 6. Average execution time for single request [ms] for various algorithms for RMSF fragmentation metric.

\begin{tabular}{l|cccc}
\hline & FA-BSC & FA-MSC & FA-kSP & FF \\
\hline no SCC & 26.4 & 29.1 & 2.6 & 1.3 \\
SCC & 10.6 & 11.5 & 2.3 & 1.3 \\
\hline
\end{tabular}

(B-SChs) selection was proposed which allowed for selecting promising candidate SChs for allocation that minimize the fragmentation. We developed and introduced a fragmentationaware with bordering super-channels (FA-BSC) algorithm which considers both spatial continuity, and the case of relaxation of such constraint as well. FA-BSC leverage proposed fragmentation metrics and the concept of B-SChs to minimizes network fragmentation, and thus the blocking probability. According to the results, FA-BSC yields better results when compared to reference algorithms. Especially, the selection of B-SChs provides better results when compared to the selection of corresponding number of other candidate SChs without specified strategy. Thus, proper selection of candidate SChs in fragmentation-aware algorithms may finally improve network fragmentation, and in consequence, bandwidth blocking probability.

In future work, we plan to focus more on the impact of various fiber technologies, such as, multi-core fiber vs. multi-mode fiber, and investigate to what extent their differences in the physical layer impact the fragmentation metrics and fragmentationaware dynamic algorithms.

\section{FUNDING}

This work was supported by National Science Centre, Poland under Grant 2017/27/B/ST7/00888.

\section{REFERENCES}

1. P. J. Winzer, "Spatial multiplexing in fiber optics: The 10x scaling of metro/core capacities," Bell Labs Tech. J. 19, 22-30 (2014).

2. G. M. Saridis, D. Alexandropoulos, G. Zervas, and D. Simeonidou, "Survey and evaluation of space division multiplexing: From technologies to optical networks," IEEE Commun. Surv. \& Tutorials 17, 2136-2156 (2015).

3. M. Klinkowski, P. Lechowicz, and K. Walkowiak, "Survey of resource allocation schemes and algorithms in spectrally-spatially flexible optical networking," Opt. Switch. Netw. 27, 58-78 (2018).

4. M. Jinno, H. Takara, B. Kozicki, Y. Tsukishima, Y. Sone, and S. Matsuoka, "Spectrum-efficient and scalable elastic optical path network: architecture, benefits, and enabling technologies," IEEE Commun. Mag. 47, 66-73 (2009).

5. B. C. Chatterjee, S. Ba, and E. Oki, "Fragmentation problems and management approaches in elastic optical networks: A survey," IEEE Commun. Surv. \& Tutorials 20, 183-210 (2018).

6. K. Walkowiak, M. Klinkowski, and P. Lechowicz, "Dynamic routing in spectrally spatially flexible optical networks with back-to-back regeneration," IEEE/OSA J. Opt. Commun. Netw. 10, 523-534 (2018).

7. P. S. Khodashenas, J. M. Rivas-Moscoso, D. Siracusa, F. Pederzolli, B. Shariati, D. Klonidis, E. Salvadori, and I. Tomkos, "Comparison of spectral and spatial super-channel allocation schemes for SDM networks," J. Light. Technol. 34, 2710-2716 (2016).

8. R. Rumipamba-Zambrano, F.-J. Moreno-Muro, J. Perelló, P. PavónMarin̄o, and S. Spadaro, "Space continuity constraint in dynamic flexgrid/SDM optical core networks: An evaluation with spatial and spectral super-channels," Comput. Commun. 126, 38 - 49 (2018).

9. M. Yang, Q. Wu, and Y. Zhang, "Joint assignment of spatial granularity, routing, modulation, and spectrum in SDM-EONs: Minimizing the network CAPEX considering spectrum, WSS, and laser resources," J. Light. Technol. 36, 4153-4166 (2018).
10. D. M. Marom and M. Blau, "Switching solutions for WDM-SDM optical networks," IEEE Commun. Mag. 53, 60 - 68 (2015).

11. P. Lechowicz, M. Tornatore, A. Włodarczyk, and K. Walkowiak, "Fragmentation metrics in spectrally-spatially flexible optical networks," in 2019 23rd Conference on Optical Network Design and Modeling (ONDM), (2019).

12. D. Amar, E. Le Rouzic, N. Brochier, J.-L. Auge, C. Lepers, and N. Perrot, "Spectrum fragmentation issue in flexible optical networks: analysis and good practices," Photonic Netw. Commun. 29, 230-243 (2015).

13. S. Fujii, Y. Hirota, H. Tode, and K. Murakami, "On-demand spectrum and core allocation for reducing crosstalk in multicore fibers in elastic optical networks," IEEE/OSA J. Opt. Commun. Netw. 6, 1059-1071 (2014).

14. H. Tode and Y. Hirota, "Routing, spectrum, and core and/or mode assignment on space-division multiplexing optical networks," IEEE/OSA J. Opt. Commun. Netw. 9, A99-A113 (2017).

15. L. Liu, Z. Zhu, and S. J. Ben Yoo, "3D elastic optical networks in temporal, spectral, and spatial domains with fragmentation-aware rssma algorithms," in 2014 The European Conference on Optical Communication (ECOC), (2014), pp. 1-3.

16. Y. Zhao and J. Zhang, "Crosstalk-aware cross-core virtual concatenation in spatial division multiplexing elastic optical networks," Electron. Lett. 52, 1701-1703 (2016).

17. Y. Zhao et al., "Crosstalk-aware spectrum defragmentation based on spectrum compactness in space division multiplexing enabled elastic optical networks with multicore fiber," IEEE Access 6, 15346-15355 (2018)

18. S. Sugihara et al., "Dynamic resource allocation for immediate and advance reservation in space-division-multiplexing-based elastic optical networks," IEEE/OSA J. Opt. Commun. Netw. 9, 183-197 (2017).

19. S. Trindade and N. L. S. da Fonseca, "Proactive fragmentation-aware routing, modulation format, core, and spectrum allocation in EON-SDM," in ICC 2019 - 2019 IEEE International Conference on Communications (ICC), (2019), pp. 1-6.

20. F. Yousefi and A. G. Rahbar, "Novel fragmentation-aware algorithms for multipath routing and spectrum assignment in elastic optical networksspace division multiplexing (EON-SDM)," Opt. Fiber Technol. 46, 287296 (2018)

21. G. Meloni, F. Fresi, M. Imran, F. Paolucci, F. Cugini, A. D’Errico, L. Giorgi, T. Sasaki, P. Castoldi, and L. Pot, "Software-defined defragmentation in space-division multiplexing with quasi-hitless fast core switching," J. Light. Technol. 34, 1956-1962 (2016).

22. P. Wright, M. C. Parker, and A. Lord, "Minimum- and maximum-entropy routing and spectrum assignment for flexgrid elastic optical networking," IEEE/OSA J. Opt. Commun. Netw. 7, A66-A72 (2015).

23. Z. Ye, A. N. Patel, P. N. Ji, and C. Qiao, "Root mean square (RMS) factor for assessing spectral fragmentation in flexible grid optical networks," in 2014 OptoElectronics and Communication Conference and Australian Conference on Optical Fibre Technology, (2014), pp. 357-358.

24. C. Rottondi, M. Tornatore, and G. Gavioli, "Optical ring metro networks with flexible grid and distance-adaptive optical coherent transceivers," Bell Labs Tech. J. 18, 95-110 (2013).

25. P. S. Khodashenas, J. M. Rivas-Moscoso, D. Siracusa, F. Pederzolli, B. Shariati, D. Klonidis, E. Salvadori, and I. Tomkos, "Comparison of spectral and spatial super-channel allocation schemes for SDM networks," J. Light. Technol. 34, 2710-2716 (2016).

26. A. Horota, L. Reis, G. Figueiredo, and N. L. S. da Fonseca, "Routing and spectrum assignment algorithm with most fragmented path first in elastic optical networks," in 2015 7th IEEE Latin-American Conference on Communications (LATINCOM), (2015), pp. 1-6.

27. J. Shen, J. Chen, and Y. Sun, "Fragmentation aware routing and spectrum assignment algorithm for elastic optical networks," in TENCON 2015 - 2015 IEEE Region 10 Conference, (2015), pp. 1-4.

28. K. Walkowiak, P. Lechowicz, M. Klinkowski, and A. Sen, "ILP modeling of flexgrid sdm optical networks," in 2016 17th International Telecommunications Network Strategy and Planning Symposium (Networks), (2016), pp. 121-126. 